\title{
Magnesium infusion for refractory non-surgical pheochromocytoma crisis
}

\author{
Chelsea K Krueger PharmD, Kimberly A Turner MPAS, PA-C, Raymond A Moreno AG-ACNP, \\ Biosha B Jones, Olakunle Idowu MD
}

\begin{abstract}
Pheochromocytoma crisis is a rare medical emergency characterized by severe hypertension, shock, and multiorgan failure. Despite its widespread availability, low cost, and wide therapeutic index, magnesium sulfate has been utilized primarily as an adrenergic antagonist during pheochromocytoma resection. This case report describes a 57-year-old man with recurrent nonsurgical pheochromocytoma crisis refractory to adrenergic antagonism. Initiation of a magnesium sulfate continuous infusion provided rapid hemodynamic control in the setting of inoperable disease. This case highlights the role and dosing of magnesium sulfate in the management of pheochromocytoma crisis.
\end{abstract}

Keywords: Pheochromocytoma, magnesium sulfate, hypertension, hemodynamics, adrenal gland neoplasm

\section{INTRODUCTION}

Pheochromocytoma is a catecholamine secreting neoplasm of the adrenal medulla with an annual incidence of $500-1,100$ cases in the United States. ${ }^{1}$ Extremely high levels of circulating catecholamines may precipitate a pheochromocytoma crisis characterized by severe hypertension, shock, and multiorgan failure. Mortality rates as high as $85 \%$ have been reported in patients who experience a crisis, and traditional pharmacologic agents are suboptimal due to limited efficacy, slow onset, and risk of hemodynamic compromise. .,3 $^{2,3}$

Magnesium produces direct arteriolar dilation through calcium channel blockade, inhibition of catecholamine release, and antagonism of catecholamine receptors. ${ }^{4,5}$ Despite its widespread availability,

Corresponding author: Olakunle Idowu

Contact Information: Oldowu@mdanderson.org

DOI: 10.12746/swrccc.v7i30.531 low cost, and wide therapeutic index, magnesium sulfate has been used primarily during the perioperative period of pheochromocytoma resection. ${ }^{6}$ Only one case series reported successful blood pressure reduction during pheochromocytoma crisis, and there are minimal data to guide dosing and management in this high risk patient population. ${ }^{3}$ Here we report a case of pheochromocytoma crisis refractory to adrenergic blockade that was successfully managed with magnesium sulfate infusion.

\section{CASE}

A 57-year-old man with a history of paraganglioma and pheochromocytoma who presented to the Emergency Department (ED) with weakness and light-headedness. The primary tumor was resected upon diagnosis 5 years prior but subsequently reoccurred with retroperitoneal and osseous disease recurrence. He was initiated on an investigational protocol with pembrolizumab 6 months prior which he reported caused blood pressure swings. He noted progressive dyspnea on exertion over two weeks and 
abnormally labile blood pressures exacerbated by poor compliance with home medications.

Initial vital signs in the ED included blood pressure $227 / 139 \mathrm{mmHg}$ and heart rate 110 beats per minute. He was diaphoretic and had an S4 gallop. Laboratory studies revealed non-oliguric acute kidney injury with a serum creatinine of $180 \mu \mathrm{mol} / \mathrm{L}$ elevated from baseline of $106 \mu \mathrm{mol} / \mathrm{L}$. Free normetanephrine was elevated at $84 \mathrm{nmol} / \mathrm{L}$. A 12-lead electrocardiogram demonstrated t-wave inversion due to demand ischemia. A two-dimensional transthoracic echocardiogram revealed moderate concentric left ventricular hypertrophy. In the ED, he received phentolamine $1 \mathrm{mg}$ IV bolus which produced a $60 \%$ decrease in mean arterial pressure. He was admitted to the intensive care unit (ICU) for hypertensive emergency secondary to unresectable pheochromocytoma.

In the ICU he became hypertensive and tachycardic again and was restarted on his home regimen of doxazosin $4 \mathrm{mg}$ daily and propranolol $20 \mathrm{mg}$ three times daily (TID). A nicardipine infusion was started with titration parameters to a target systolic blood pressure (SBP) of $140-160 \mathrm{mmHg}$. Over the next 2 days he failed multiple attempts to wean off nicardipine, despite increasing doxazosin to $8 \mathrm{mg}$ daily and propranolol to $30 \mathrm{mg}$ TID.

On day 3 he received a continuous infusion magnesium sulfate for hypertension refractory to alphaand beta-adrenergic blockade. Baseline serum magnesium prior to infusion was $0.86 \mathrm{mmol} / \mathrm{L}$. A loading dose of $4 \mathrm{~g}$ was infused intravenously over 2 hours, followed by a $1 \mathrm{~g} / \mathrm{h}$ infusion. Serum magnesium levels and deep tendon reflexes were checked every 6 hours. Blood pressure decreased from $182 / 118 \mathrm{mmHg}$ to $117 / 74 \mathrm{mmHg}$ following completion of the loading dose. During the maintenance infusion, SBP remained below the target of $160 \mathrm{mmHg}$ (range 112-146 $\mathrm{mmHg}$ ). The infusion was stopped after 11 hours for an asymptomatic elevated serum magnesium level of $2.02 \mathrm{mmol} / \mathrm{L}$. Thirteen grams of magnesium sulfate were infused prior to discontinuation. Blood pressure readings were noted to be relatively stable for the duration of the infusion (Figure 1) and for the following 24 hours.

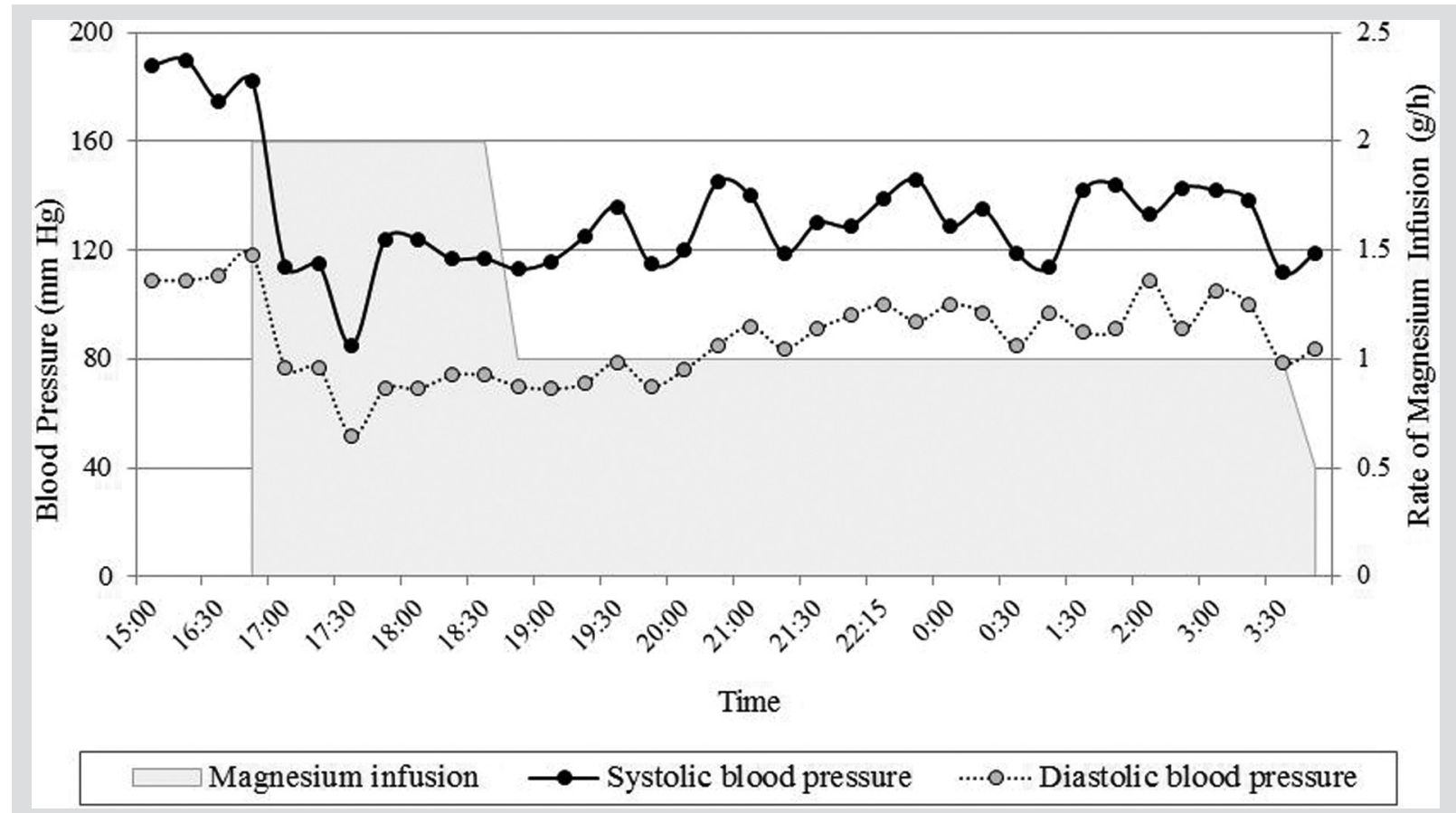

Figure 1. Blood pressure values during continuous infusion of magnesium sulfate. 
Magnesium infusion was not resumed due to concerns for accumulation and hypermagnesemia. Blood pressure was subsequently managed by titrating doxazosin to $8 \mathrm{mg}$ twice daily and propranolol to $40 \mathrm{mg}$ TID. Nicardipine infusion was not required, and nifedipine $90 \mathrm{mg}$ by mouth daily was started. The patient was transferred out of the ICU on day 5 and discharged from the hospital on day 8 without subsequent readmission.

\section{Discussion}

This report illustrates the utility of magnesium infusion for the management of pheochromocytoma crisis in a patient with metastatic, nonresectable disease. Pheochromocytomas and paragangliomas are rare catecholamine-secreting neuroendocrine tumors that arise from the adrenal medulla and extra-adrenal tissues, respectively. ${ }^{5}$ Early diagnosis is often prompted by the classic symptom triad of headache, palpitations, and diaphoresis. ${ }^{7}$ The presentation of pheochromocytoma crisis varies but is associated with uncontrolled hypertension that may progress to circulatory failure, encephalopathy, pulmonary edema, and multiorgan failure. ${ }^{8-9}$

Management of pheochromocytoma crisis focuses on pharmacologic control of hemodynamics, restoration of blood volume, and timely surgical resection. ${ }^{4-6}$ In patients with unresectable disease, management of a crisis includes alpha-adrenergic blockade and intravascular volume repletion with adjunctive calcium channel and beta-adrenergic blockade. ${ }^{5,10}$ Magnesium has a well-established perioperative role during pheochromocytoma resection, but its use in nonsurgical crisis has been examined only in one small case series. ${ }^{3-6}$ Magnesium inhibits catecholamine release from the adrenal medulla and directly antagonizes adrenergic receptors to produce arteriolar vasodilation. ${ }^{2}$ Blockade of catecholamine surges during surgical resection makes it an important component of intraoperative management. In addition, magnesium antagonizes L-type calcium channels and sodium-potassium-ATPase pumps, producing antiarrhythmic and membrane stabilizing properties. ${ }^{2,3}$
Despite its widespread availability, low cost, and wide therapeutic index, the optimal dose for magnesium infusions in pheochromocytoma is not well established. Large 40-60 mg/kg intraoperative loading doses have been administered safely followed by $2 \mathrm{~g} / \mathrm{h}$ maintenance infusions. ${ }^{11} \mathrm{~A}$ high intraoperative serum magnesium level of 1.97 to $3.99 \mathrm{mmol} / \mathrm{L}$ has even been proposed as a target range for maximal effect. ${ }^{11,12}$ Close monitoring is warranted, however, as neuromuscular and cardiac toxicity may be seen at serum levels higher than $2.96 \mathrm{mmol} / \mathrm{L}$. The appropriate dose for nonsurgical crisis is less established. A case series of 3 patients with refractory nonsurgical pheochromocytoma crisis reported benefit when magnesium was given as a 2-4 g loading dose followed by a $1-2 \mathrm{~g} / \mathrm{h}$ continuous infusion in conjunction with alpha-adrenergic blockade. ${ }^{3}$ No adverse effects were seen in 2 patients, and 1 patient reportedly maintained a serum magnesium level of 2.47-3.0 mmol/L. In the patient who experienced an adverse effect, magnesium infusion was held for weakness that occurred at a serum magnesium level of $3.91 \mathrm{mmol} / \mathrm{L}$. Our threshold for holding therapy was more conservative and led to discontinuation of infusion at a serum level of $2.02 \mathrm{mmol} / \mathrm{L}$ despite a lack of symptoms. The aggressive dosing adopted in that case series likely reflects the more severe manifestations of pheochromocytoma crisis in those 3 patients.

Magnesium infusion is a temporary measure for the management of pheochromocytoma crisis, as long-term use is complicated by potential magnesium toxicity and the need for intravenous access. Concurrent alpha-adrenergic antagonists (e.g., doxazosin, phenoxybenzamine, or phentolamine) should be optimized during the initial crisis with beta-adrenergic antagonists given to control tachycardia. ${ }^{5,6,10}$ Phentolamine and doxazosin both act as competitive antagonists and may have diminished effect in patients with massive adrenergic stimulation. ${ }^{5}$ Phenoxybenzamine is a noncompetitive antagonist, but its slow onset of action limits use as monotherapy. ${ }^{5}$ As shown in this case, patients with nonsurgical pheochromocytoma crisis may achieve symptom resolution with adjunctive magnesium infusion. Magnesium infusion should be continued 
as alpha-adrenergic antagonists are optimized, and patients should be closely monitored for signs of magnesium toxicity.

\section{Conclusion}

In summary, we present a patient with pheochromocytoma crisis refractory to alpha-adrenergic antagonism. Magnesium sulfate continuous infusion provided rapid hemodynamic control and represents a safe, cost-effective therapy for the initial management of nonsurgical pheochromocytoma crisis.

Article citation: Krueger CK, Turner KA, Moreno RA, Jones BB, Idowu O. The Southwest Respiratory and Critical Care Chronicles 2019; 7(30):47-50

From: University of Texas MD Anderson Cancer Center Division of Pharmacy (CKK); Texas Tech University Health Sciences Center School of Pharmacy (CKK); University of Texas MD Anderson Cancer Center Department of Critical Care (KAT, RAM, BBJ, OI).

Submitted: $2 / 23 / 2019$

Accepted: 4/30/2019

Reviewer: Camilo Pena MD

Conflicts of interest: none

This work is licensed under a Creative Commons Attribution-ShareAlike 4.0 International License.

\section{REFERENCES}

1. O'Riordan JA. Pheochromocytomas and anesthesia. Int Anesthesiol Clin 1997;35:99-127.

2. Herroeder S, Schönherr ME, De Hert SG, et al. Magnesium: essentials for anesthesiologists. Anesthesiology 2011; 114:971-993.

3. James MF, Cronjé L. Pheochromocytoma crisis: the use of magnesium sulfate. Anesth Analg 2004;99:680-686.

4. Dubé L, Granry JC. The therapeutic use of magnesium in anesthesiology, intensive care and emergency medicine: a review. Can J Anaesth 2003;50:732-746.

5. Domi R, Laho H. Management of pheochromocytoma: old ideas and new drugs. Niger J Clin Pract 2012;15:253-257.

6. Lord MS, Augoustides JG. Perioperative management of pheochromocytoma: focus on magnesium, clevidipine, and vasopressin. J Cardiothorac Vasc Anesth 2012;26:526-531.

7. Werbel SS, Ober KP. Pheochromocytoma: update on diagnosis, localization, and management. Med Clin North Am 1995;79:131-153.

8. Kizer JR, Koniaris LS, Edelman JD, et al. Pheochromocytoma crisis, cardiomyopathy, and hemodynamic collapse. Chest 2000;118:1221-1223.

9. Bergland BE. Pheochromocytoma presenting as shock. Am J Emerg Med 1989;7:44-48.

10. Shah MH, Goldner WS, Halfdanarson TR, et al. NCCN guidelines insights: neuroendocrine and adrenal tumors version 2.2018. J Natl Compr Canc Netw 2018;16:693-702.

11. James MF. Use of magnesium sulphate in the anaesthetic management of pheochromocytoma: a review of 17 anaesthetics. Br J Anaesth 1989;62:6161-6123.

12. Minami T, Adachi T, Fukuda K. An effective use of magnesium sulfate for intraoperative management of laparoscopic adrenalectomy for pheochromocytoma in a pediatric patient. Anesth Analg 2002;95:1243-1244. 